\title{
Deriving an Emergent Relational Schema from RDF Data
}

\author{
Minh-Duc Pham $₫$ \\ m.d.pham@vu.nl
}

\author{
Linnea Passing
passing@in.tum.de \\ Linnea Passing
passing@in.tum.de
}

$\triangle$ Vrije Universiteit Amsterdam, The Netherlands
${ }^{\square}$ Technische Universität München, Germany

$\diamond$ OpenLink Software, UK

\author{
Peter Boncz ${ }^{\odot}$ \\ boncz@cwi.nl
}

\begin{abstract}
We motivate and describe techniques that allow to detect an "emergent" relational schema from RDF data. We show that on a wide variety of datasets, the found structure explains well over $90 \%$ of the RDF triples. Further, we also describe technical solutions to the semantic challenge to give short names that humans find logical to these emergent tables, columns and relationships between tables. Our techniques can be exploited in many ways, e.g., to improve the efficiency of SPARQL systems, or to use existing SQL-based applications on top of any RDF dataset using a RDBMS.
\end{abstract}

\section{Categories and Subject Descriptors}

H.3 [Information Storage and Retrieval]: Miscellaneous

\section{Keywords}

RDF; Structure recognition; Relational schema

\section{INTRODUCTION}

RDF is the data model for the Semantic Web and Linked Open Data. It represents data as collection of <subject, property, object $>$ triples. By providing flexibility for users to represent and evolve data without the need for a prior schema - sometimes called the "schema last" approach and identifying properties and (references to) subjects uniformly using URIs, RDF has been gaining ground as the standard for global data exchange and interoperability, recently through the popularization of micro-formats such as $\mathrm{RDFa}$, which are increasingly used embedded in web pages. This creates a need for database technologies that can query large amounts of RDF efficiently with SPARQL or $S Q L$.

SQL-speaking relational database systems (RDBMS's) require to declare a schema upfront ("schema first") and can only store and query data that conforms to this schema. RDF systems typically rely on a "triple-store" architecture, which store all data in a single table containing S, P and

Copyright is held by the International World Wide Web Conference Committee (IW3C2). IW3C2 reserves the right to provide a hyperlink to the author's site if the Material is used in electronic media. $W W W$ 2015, May 18-22, 2015, Florence, Italy. ACM 978-1-4503-3469-3/15/05.

http://dx.doi.org/10.1145/2736277.2741121.
0 (subject, property, object) columns ${ }^{1}$. SQL systems tend to be more efficient than triple stores, because the latter need query plans with many self-joins - one per SPARQL triple pattern. Not only are these extra joins expensive, but because the complexity of query optimization is exponential in the amount of joins, SPARQL query optimization is much more complex than SQL query optimization. As a result, large SPARQL queries often execute with a suboptimal plan, to much performance detriment. RDBMS's can further store data efficiently e.g. using advanced techniques such as column-wise compression, table partitioning, materialized views and multi-dimensional data clustering. These techniques require insight in the (tabular) structure of the dataset and have so far not been applicable to RDF stores.

Semantic Web technology has its roots in Artificial Intelligence and knowledge representation, and we think it is seldom realized that its notion of "schema" in the term "schema last" differs from the corresponding "schema" notion in "schema first" for relational technology. Semantic Web schemas - ontologies and vocabularies - are intended to allow diverse organizations to consistently denote certain concepts in a variety of contexts. In contrast, relational schemas describe the structure of one database (=dataset), designed without regard for reuse in other databases.

Our work shows that actual RDF datasets exhibit (i) a very partial use of ontology classes and (ii) subjects share triples with properties from classes defined in multiple ontologies. To illustrate, (i) in the crawled WebDataCommons data there is information on less than a third of the ontology class properties in the actual triples, and (ii) we find in DBpedia that each subject combines information from more than eight different ontology classes on average. As such, when analyzing the actual structure of RDF datasets by observing which combinations of properties typically occur together with a common subject (called "Characteristic Sets" of properties [14]), any single ontology class tends to be a poor descriptor. Knowledge of the actual structure of a dataset is essential for RDBMS's to be able to store and query data efficiently. Our work allows RDF stores to automatically discover this actual structure, which we call the emergent relational schema. The emergent relational schema enables to internally store RDF data more like relational tables, allowing SPARQL query execution to use less self-joins, which also reduces the complexity of query opti-

\footnotetext{
${ }^{1}$ With "triple-store" we mean RDF or graph stores that use any data structure, be it a graph edge-list, B-tree, hash map, etc. that stores individual triples (or quads), or graph edges without exploiting their connection structure.
} 
mization [10]. Note that not all triples in a RDF dataset need to conform to this relational schema for these techniques to be effective, as long as the great majority does. Hence, RDF remains as flexible as ever in emergent relational schema aware systems.

There is also a usability advantage if the actual structure of an RDF dataset would be conveyed to a human user. A common problem when posing SPARQL queries is that queries come back empty if properties that one expects to occur given ontology knowledge, turn out not to be present in the data. Or, one may lack any ontology knowledge and thus have little to go by when querying. However, automatically deriving a human-friendly relational schema from a RDF dataset introduces additional challenges to recognizing its structure, since all the schema elements (tables, columns) should get correct and short labels, and the emergent relational schema must be compact to be understandable.

Our work presents a self-tuning algorithm that surmounts this challenge, which we tested on a wide variety of RDF datasets. We integrated our techniques in two open-source state-of-the art data management systems: the well-known RDF store Virtuoso and the MonetDB DBMS. The RDF bulkload in MonetDB now offers efficient SQL access to any $\mathrm{RDF}$ dataset via its emergent relational schema, allowing the wealth of SQL-based applications over ODBC and JDBC (e.g. Business Intelligence tools like Tableau) to be used. By doing so we are enriching RDBMS's with web standards, because these relational tables, columns and foreign key (FK) constrains are identifiable using ontology-based URIs, and even the primary key values and foreign key values themselves are URIs (RDF subjects resp. non-literal objects). As such, our work is a bridge between the Semantic Web and RDBMS's, enriching both worlds.

Contributions of our work are the following:

1) We identify an important difference between Semantic Web schema information (describing a knowledge universe) and relational schemas (describing one dataset), and argue that both should be available to data stores and their users.

2) We present methods for detecting the basic table structure and the relationships between them from an RDF dataset, and propose several approaches to semantically and structurally optimize the relational schema to make it compact.

3) We present techniques to assign human-friendly names to tables/columns and their FK relationships.

4) Our experiments on a wide variety of RDF datasets show that (i) over $90 \%$ of the triples in these conform to a compact emergent relational schema, (ii) our algorithms are efficient and can be executed during RDF bulk load with little overhead, (iii) RDF stores can improve both query optimization and execution by exploiting the emergent relational schema, and (iv) we illustrate with a user survey that the short human-readable labels we find have good quality.

\section{EMERGING A RELATIONAL SCHEMA}

The five steps of our emergent relational schema algorithm detect something akin to a UML class diagram by analyzing Characteristic Sets (CS's) [14] in an RDF input dataset:

1. Basic CS Discovery. We discover all occurring CS's from a bulk-loaded SPO table and count their frequencies. Then, we analyze properties in each CS that are not literals, i.e. refer to URIs (and hence to other CS's) in order to explore the relationships between CS's.
2. CS Labeling. We assign class, attribute and relationship labels (human-understandable names) to the recognized CS's using multiple methods.

3. CS Merging. We merge CS's that are semantically or structurally similar to each other, with the purpose of making the schema more compact. We re-run Steps 2 and 3 iteratively in order to automatically tune the similarity threshold parameter $\tau_{\text {sim }}$ to the nature of the dataset.

4. Schema Filtering. We filter low frequency CS's, but make sure to conserve highly referenced CS's (akin to relational "dimension tables"). As reference relationships can be indirect (via via) we use a PageRank-like algorithm to count how often referenced each CS is. We also filter out CS properties that are too sparsely populated.

5. Instance Filtering. We filter out instances (rows) to increase literal type homogeneity, and filter out individual triples to eliminate erroneously multi-valued attributes, and to improve foreign-key cardinality homogeneity.

The "class diagram" where each merged-CS that survived filtering is a class, is represented as a relational schema consisting of tables and foreign key relationships. Each class becomes one table, and its properties its columns, but relationships and multi-valued attributes lead to additional tables. Properties for which multiple literal types occur frequently, are represented by multiple table columns. The $<10 \%$ triples that do not fit this schema remain stored in a separate SPO table. We now discuss the five steps in detail.

\subsection{Basic CS Discovery}

Given an RDF dataset $R$, the Characteristic Set of a subject $s$ is defined as $c s(s)=\{p \mid \exists o:(s, p, o) \in R\}$ [14].

We first identify the basic set of CS's by analyzing all triples stored in an RDF table in SPO order. Such table is produced by a standard bulk load employed by triple stores. While loading the triples into this representation, the URIs get encoded in a dictionary, such that columns S, P and $\mathrm{O}$ are not URI strings, but integers called object identifiers (OIDs) pointing into this dictionary. This is a standard technique. These integer OIDs form a dense domain starting at 0 .

We now make a single pass through the SPO table and fill a hash map where the key is the set of OIDs of properties that co-occur for each subject. Note that due to the SPO ordering these are easily found as the P's of consecutive SPO triples with equal S. The key of the hash map is the offset in the SPO table where the CS first occurs. Its hash is computed by XOR-ing the hashes of all P's (which are OIDs). The insert-order in the hash table (starting at 1) provides us with a dense numeric OID for each CS. Further, we remember in an array indexed by $\mathrm{S}$ which stores such CS-OIDs, to which CS each subject belongs (this array is part of the URI dictionary). Note that not all URIs in the dictionary may occur as a subject in the SPO table, for which case this array is initialized with zeros. After making the single pass over the SPO table, we will have all occurring basic $C S$-s in the hash map, and we also keep an occurrence count there.

Further we make a second pass over the SPO table, where we look at type information. For each triple with a literal object, we maintain a histogram of literal-type occurrences per property in a second hash map with key [P, type] and a count value. For each triple that is a non-literal, on the other hand, we look up to which CS its subject belongs ( $\operatorname{srcCS})$ and to which its object (dstCS) - this can be done efficiently using the array mentioned before. If there is a 


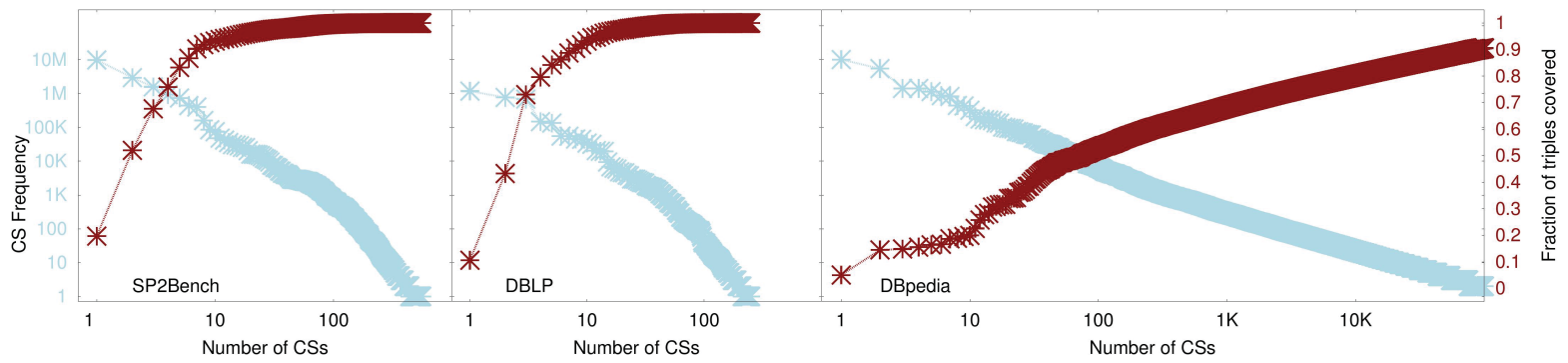

Figure 1: CS Frequency (light blue) vs. Cumulative number of covered triples (dark red)

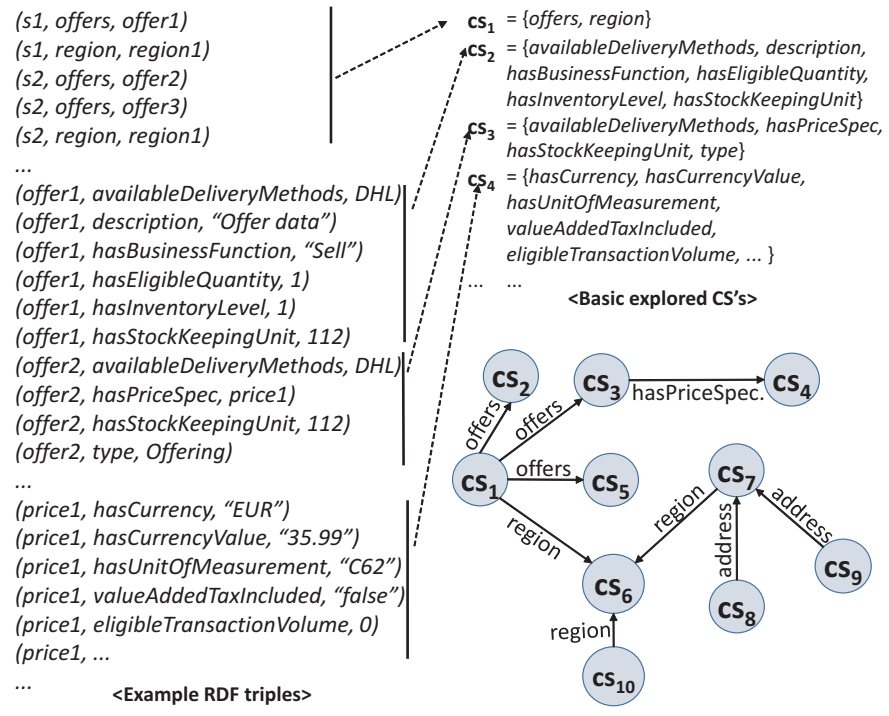

Figure 2: Example of basic CS's and their relationships

dstCS, we maintain another histogram stored in a third hash map with as key [srcCS, P, dstCS] and a count value. This histogram records how often basic-CS's refer to each other and over which property (relationship statistics).

These algorithms are all simple and obviously linear in average-case complexity, therefore we omit a listing or further analysis. Figure 2 shows an example of the found basic CS and their relationships after the exploration process.

Diversity of the basic CS's. Table 1 shows statistics on the basic CS's and their properties for the synthetic RDF benchmark datasets $\mathrm{LUBM}^{2}, \mathrm{SP}_{2} \mathrm{~B}^{3}$, and $\mathrm{BSBM}^{4}$, the originally relational datasets converted to $\mathrm{RDF}$ MusicBrainz ${ }^{5}$, EuroStat ${ }^{6}$, and $\mathrm{DBLP}^{7}, \mathrm{PubMed}^{8}$ and the native RDF datasets WebDataCommons 9 ("WebData.") and DBpedia ${ }^{10}$. The number of basic CS's can vary significantly regardless the number of input triples. If one would naively propose to store RDF data using a separate relational table for each

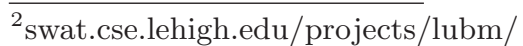

${ }^{3}$ dbis.informatik.uni-freiburg.de/forschung/projekte/SP2B/

${ }^{4}$ wifo5-03.informatik.uni-mannheim.de/bizer/

berlinsparqlbenchmark/

${ }^{5}$ linkedbrainz.c4dmpresents.org/data/

musicbrainz_ngs_dump.rdf.ttl.gz

${ }^{6}$ eurostat.linked-statistics.org

${ }^{7}$ gaia.infor.uva.es/hdt/dblp-2012-11-28.hdt.gz

8 www.ncbi.nlm.nih.gov/pubmed

${ }^{9} \mathrm{~A} 100 \mathrm{M}$ triple file of webdatacommons.org

${ }^{10}$ dbpedia.org - we used v3.9
}

\begin{tabular}{|c|c|c|c|c|c|}
\hline Datasets & \#triples* & \#CS's & $\begin{array}{l}\text { \#CS's to } \\
\text { cover } 90 \%\end{array}$ & $\begin{array}{c}\text { Avg. } \\
\text { \#prop. }\end{array}$ & $\begin{array}{l}\text { \#multi-type } \\
\text { properties }\end{array}$ \\
\hline LUBM & $100 \mathrm{M}$ & 17 & 7 & 5.71 & 0 \\
\hline BSBM & $100 \mathrm{M}$ & 49 & 14 & 12.61 & 0 \\
\hline SP2Bench & $100 \mathrm{M}$ & 554 & 7 & 9.8 & 0 \\
\hline synthetic & \multicolumn{5}{|c|}{ data created by benchmark data generator } \\
\hline MusicBrainz & $179 \mathrm{M}$ & 27 & 10 & 4.7 & 0 \\
\hline EuroStat & $70 \mathrm{~K}$ & 44 & 8 & 7.77 & 0 \\
\hline DBLP & $56 \mathrm{M}$ & 249 & 8 & 13.70 & 0 \\
\hline PubMed & $1.82 \mathrm{~B}$ & 3340 & 35 & 19.27 & 0 \\
\hline relational & \multicolumn{5}{|c|}{$R D F$ data from a relational database dump } \\
\hline WebData. & $90 \mathrm{M}$ & 13354 & 930 & 7.94 & 551 \\
\hline DBpedia & $404 \mathrm{M}$ & 439629 & 85922 & 24.36 & 1507 \\
\hline
\end{tabular}

Table 1: Statistics on basic CS's.

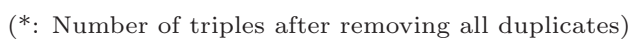

basic CS, we now see that a complex RDF dataset like DBpedia would lead to an unacceptable number of small tables. As we can also see that while most of the datasets have a single literal type for each CS property, DBpedia and WebDataCommons have many properties with more than one literal type in its object values (i.e., multi-type properties), so native datasets appear to be both complex and "dirty".

Data coverage by basic CS's. Figure 1 shows the frequencies and the cumulative number of triples covered by the basic CS's sorted by their frequencies, for one of each kind of dataset (synthetic, relational, native). In this figure, the number of CS's needed for covering a large portion of the triples (e.g., 90\%) can be significantly different between the datasets. We show for reference in Table 1, that $90 \%$ of the synthetic benchmark datasets can be covered by using a small number of CS's (e.g., 7 for SP2Bench). Many Linked Open Data datasets originate from existing sources whose data is kept in relational databases. We see that in such datasets a few CS's can cover almost all data. However, for complex datasets originally created as RDF (native), in order to cover $90 \%$ of the triples, many CS's are needed, in case of DBpedia more than 85,000.

\subsection{CS Labeling}

When presenting humans with a relational schema, short labels should be used as aliases for machine-readable and unique URIs for naming tables, columns and relationships between tables. For assigning labels to CS's, we exploit semantic information (ontologies) as well as structural information. Because not all ontologies follow the same structure, we developed a simple vocabulary to standardize minimal aspects of an ontology, namely classes and their properties, relationships between classes, their labels, as well as the subclass hierarchy. We expressed a large set of common ontolo- 


\begin{tabular}{|c|c|c|}
\hline & mixed & partial \\
\hline dataset & $\begin{array}{c}\text { number of } \\
\text { ontology } \\
\text { classes } \\
\text { used per CS }\end{array}$ & $\begin{array}{c}\text { \%ontology } \\
\text { class } \\
\text { properties } \\
\text { used per CS }\end{array}$ \\
\hline LUBM & 1.94 & $37 \%$ \\
BSBM & 3.96 & $3 \%$ \\
SP2Bench & 4.94 & $4 \%$ \\
MusicBrainz & 3.93 & $1 \%$ \\
EuroStat & 3.14 & $84 \%$ \\
DBLP & 6.58 & $8 \%$ \\
PubMed & 4.94 & - \\
WebData. & 2.27 & $33 \%$ \\
DBpedia & 8.35 & $5 \%$ \\
\hline
\end{tabular}
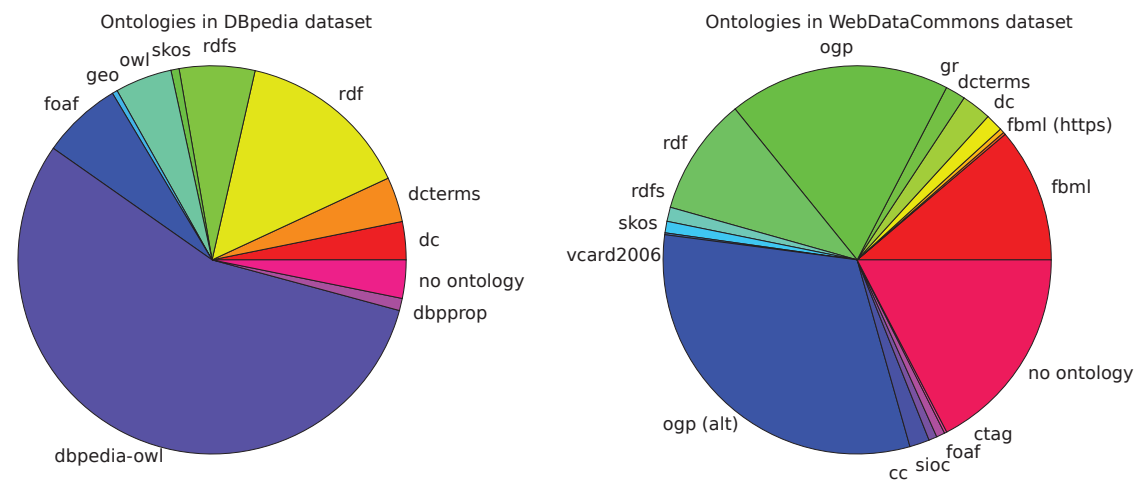

Figure 3: Partial \& mixed ontology class usage in CS's (table-left), Ontologies used in native RDF datasets (graphs-right)

\begin{tabular}{|l|r|r|}
\hline label of & \multicolumn{2}{|c|}{ subjects } \\
rdf : type & CS \% & all \% \\
\hline \hline Thing & 100 & 83 \\
Organization & 100 & 7 \\
RadioStation & $\mathbf{9 7}$ & $\mathbf{0 . 2}$ \\
Company & 1 & 4 \\
\hline
\end{tabular}

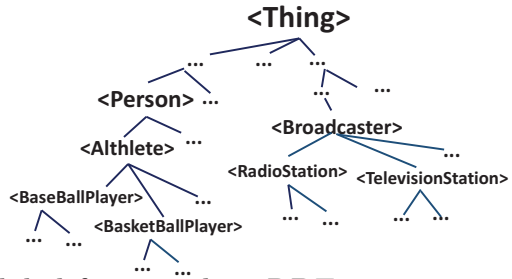

\begin{tabular}{l}
\multicolumn{1}{c|}{$\mathbf{c S}_{\mathbf{4}}$} \\
\hline dc:description \\
\hline gor:validFrom \\
\hline gor:validThrough \\
\hline gor:hasCurrency \\
\hline gor:hasCurrencyValue \\
\hline gor:hasUnitOfMeasurement \\
\hline gor:valueAddedTaxIncluded \\
\hline gor:eligibleTransactionVolume \\
(prefix gor: \\
http://purl.org/goodrelations/v1\# \\
prefix dc: \\
http://purl.org/dc/elements/1.1/)
\end{tabular}

PriceSpecification

\begin{tabular}{|l|}
\hline gor:description \\
\hline gor:name \\
\hline gor:eligibleTransactionVolume \\
\hline gor:validFrom \\
\hline gor:validThrough \\
\hline gor:hasCurrency \\
\hline gor:hasCurrencyValue \\
\hline gor:hasUnitOfMeasurement \\
\hline gor:valueAddedTaxIncluded \\
\hline gor:hasMaxCurrencyValue \\
\hline gor:hasMinCurrencyValue \\
\hline
\end{tabular}

Figure 5: Example CS vs. Ontology class

Figure 3 shows ontology class usage in the CS's and the percentage of triples corresponding to each ontology in several datasets. As shown in the graphs, each dataset contains a mix of multiple ontologies where even the most popular ontology covers less than $56 \%$ of the data. The first column in the table shows that properties from within a single $C S$ typically stem from a number of different ontologies, e.g., the average number of ontologies used in each CS in DBpedia is 8.35. We also looked at the percentage of properties of each ontology class when used in a CS. Since an ontology class may be used in multiple CS's, we compute a weighted average (where the number of subjects in a CS is the weight). The second column in the table shows this percentage to be less than $10 \%$ in most of the datasets. In other words, the datasets make only very partial usage of the properties of each ontology class. The partial usage and mixing together mean that any individual ontology class is a poor descriptor of the structure of the data. Our emergent relational schema, aims to provide a better description.

Type properties. Certain specific properties explicitly specify the concept a subject belongs to. The most common RDF property with this role is rdf:type, where the 0 of triples with this property may be the URI identifying an ontology class. Recall that our first step is to find a good UML-like class diagram for the RDF dataset, where a CS roughly corresponds to an UML class, and specifically here we are trying to find a human-friendly short name (label) for each CS. Even though we stated above that any individual ontology class is a poor descriptor for the structure of a CS, ontologies do provide valuable clues for choosing a label (name) for the CS. The subjects that are member of a CS may have different $r d f$ : type object values, this number is also variable (there can be zero such type annotations, but also multiple). To choose one, we look at the frequency of that type annotation. First, we use the global infrequent threshold $\tau_{\text {inf }}$ (e.g., $5 \%$ ) to exclude infrequent type annota-

tions to be used for finding the CS class label. For the rest, we count (i) how many subjects in the CS have it, and (ii) how many subjects in the whole dataset have it. Similar to TF/IDF [18], dividing (i) by (ii) provides a reasonable ranking to choose an appropriate ontology class. Finally, if the ontology class has label information (and this information is typically available), we then use it as the label for the CS.

We should, however, in this ranking also take into account the class hierarchy information that an ontology provides. Thus, we account for missing superclass annotations by inferring them for the purpose of this ranking. In Figure 4, if a triple in some CS has rdf:type Company, but not Organization or Thing explicitly, we still include these annotations in the ranking calculation.

In this example, "RadioStation" is chosen as its coverage of the subjects in the CS is above $\tau_{\text {inf }}(97>5)$ and its ranking score $(97 / 0.2=485)$ is the highest.

Discriminative Properties. Even if no type property is present in the CS, we can still try to match a CS to an ontology class. We compare the property set of the CS with the property sets of ontology classes using the TF/IDF similarity score [18]. This method relies on identifying "discriminative" properties, that appear in few ontology classes only, and whose occurrence in triple data thus gives a strong hint for the membership of a specific class. An example is shown in Figure 5. In this example, as $c s_{4}$ and the class PriceSpecification of the GoodRelations ontology ${ }^{11}$ share discriminative properties like gor : hasUnit0fMeasurement and gor: valueAddedTaxIncluded, PriceSpecification can be used as the label of $c s_{4}$. Detailed computation of the TF/IDFbased similarity score between a CS and an ontology class can be found in [17]. An ontology class is considered to be matching with a CS if their similarity score exceeds the sim-

${ }^{11}$ purl.org/goodrelations/ 


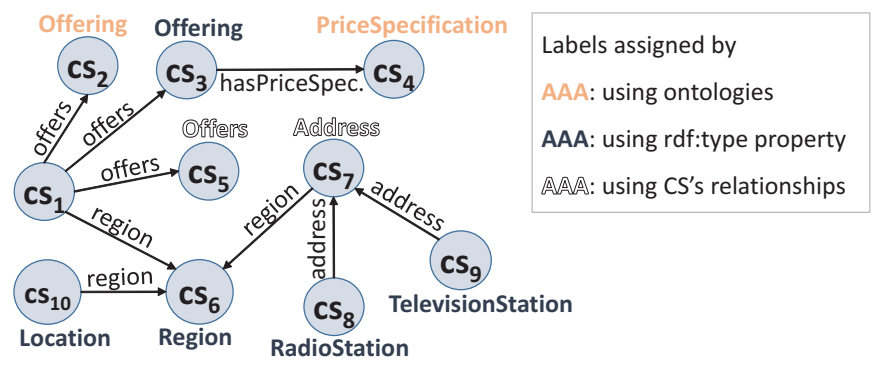

Figure 6: CS's with assigned labels

ilarity threshold $\tau_{\text {sim }}$. The ontology class correspondence of a CS, if found, is also used to find labels for properties of the CS (both for relationships and literal properties).

Relationships between CS's. If the previous approaches do not apply, we can look at which other CS's refer to a CS, and then use the URI of the referring property to derive a label. For example, a CS that is referred as Address indicates that this CS represents instances of an Address class. We use the most frequent relationship to provide a CS label. For instance, in WebDataCommons 93532 instances refer to a CS via property address and only 3 via property locatedAt. Thus, Address is chosen as the label.

URI shortening. If the above solutions cannot provide us a link to ontology information for providing attribute and relationship labels, we resort to a practical fall-back, based on the observation that often property URI values do convey a hint of the semantics. That is, for finding labels of CS properties we shorten URIs (e.g., http://purl.org/ goodrelations/v1\#offers becomes offers), by removing the ontology prefix (e.g., http://purl.org/goodrelations/ $\mathrm{v} 1 \#)$, as suggested by [15].

Note that for CS's without any ontology match or relationships with other CS's, we may find no class label candidates, in which case a synthetic default label is used. Labels are intended to help users comprehend the data, but in any case should be overridable by manual labeling. A future approach might be to look for sources on the web, such as search engines; but for the moment we prefer to keep our techniques stand-alone, as these are part of RDF bulk-load.

Figure 6 shows the labels assigned to each CS in the example dataset by using different labeling methods (e.g., the label of $c_{4}$ is assigned based on the matching between its property set and that of ontology classes, the label of $\mathrm{Cs}_{7}$ is derived from the CS's relationships, ...). In this example, $c s_{1}$ does not have any specific label as there is no sufficient information for assigning a good label to it.

\subsection{CS Merging}

After basic exploration, there may be thousands of CS's, in case of DBpedia even 100,000. This means the individual CS's have only a few subjects (=rows, in relational terms) in them, so that storing them in a relational table would incur overheads (e.g. tables not filling a disk page, large database catalog, expensive metadata lookup). Further, many of these basic CS's are very similar to each other (differing only in a few properties) and denote the same concept. When querying for that concept, one would have to formulate a UNION of many tables, which is cumbersome and also slows down queries. Finally, a relational schema with thousands of tables is just very hard to understand for

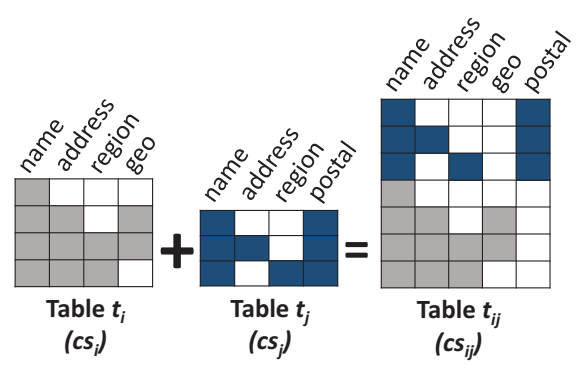

Figure 7: Example of merging CS's

humans. Therefore, the next step is to reduce the number of tables in the emergent relational schema by merging CS's, using either semantic or structural information.

Figure 7 shows an example of merging $c s_{i}$ and $c s_{j}$. We note that all subjects that fall in a basic CS do so because there exist triples for all properties in that CS, such that a relational table representing the CS would have no NULL cells. In this example, $c s_{i}$ and $c s_{j}$ represent already the results of merging other CS's (the merging process is iterative) - As shown in the figure, the number of NOT-NULL cells in table $t_{i j}$ is equal to the total number of NOT-NULL cells of the tables $t_{i}$ and $t_{j}$, however, the number of NULL cells increases due to properties not in the intersection of the two CS's becoming padded with NULLs in the merged CS.

Semantic merging. We can merge two CS's on semantic grounds when both CS class labels that we found were based on ontology information. Obviously, two CS's whose labels were taken from the same ontology class URI represent the same concept, and thus can be merged. If the labels stem from different ontology classes we can examine the class hierarchy and identify the common concept/class shared by both CS's, if any, and then justify whether these CS's are similar based on the "generality" of the concept. Here the "generality" score of a concept is computed by the percentage of instances covered by it and its subclasses among all the instances covered by that ontology (Equation 1).

$$
\begin{array}{r}
g_{\text {score }}\left(O_{c}\right)=\frac{\# \text { instances_coveredby }\left(O_{s}\right)}{\text { \#instances_coveredby_ontology }} \\
\text { where } O_{s} \text { is } O_{c} \text { or a subclass of } O_{c}
\end{array}
$$

Figure 4 showed an example of an ontology class hierarchy from DBpedia. Consider two CS labels such as RadioStation and TelevisionStation assigned by using ontology class names. By following the ontology's class hierarchy, it can be found that the corresponding classes of these labels share the same infrequent superclass Broadcaster. Therefore, these CS's can be considered as semantically similar, and could be merged with Broadcaster as new label.

More formally, there are two rules for semantic merging:

RULE 1. If an ontology class URI exists equal to the labels of both $c s_{i}$ and $c s_{j}$ then merge $c s_{i}$ and $c s_{j}$. (S1)

RuLE 2. If there exists an ontology class $O_{c}$ being an ancestor of the labels of $\mathrm{cs}_{i}$ and $\mathrm{cs}_{j}$ and $g_{\text {score }}\left(\mathrm{O}_{c}\right)$ is less than $\frac{1}{U b_{t b l}}$ then merge $c s_{i}$ and $c s_{j}$. (S2)

In $S 2, \frac{1}{U b_{t b l}}$ is used as the threshold for the generality score based on $U b_{t b l}$, the upper bound for the number of tables in the schema - which is one of the only three parameters of emergent relational schemas, see Table 2.

Figure 8 demonstrates the modifications to the explored CS's of the example dataset and their relationships when sequentially applying merging rules $S 1$ and $S 2$. Here, since $c s_{2}$ 

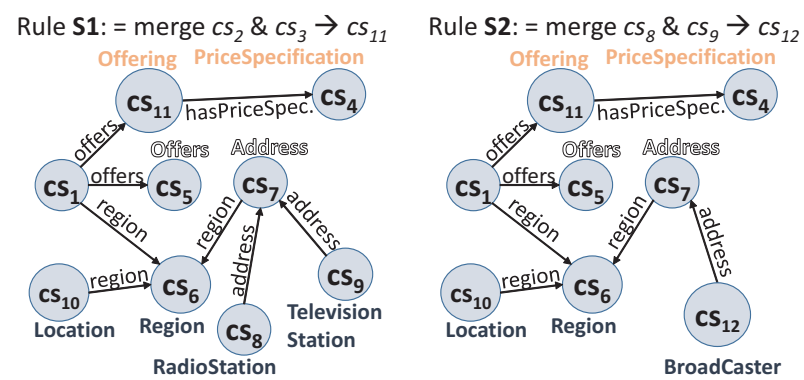

Figure 8: Example of merging CS's by using rules S1, S2
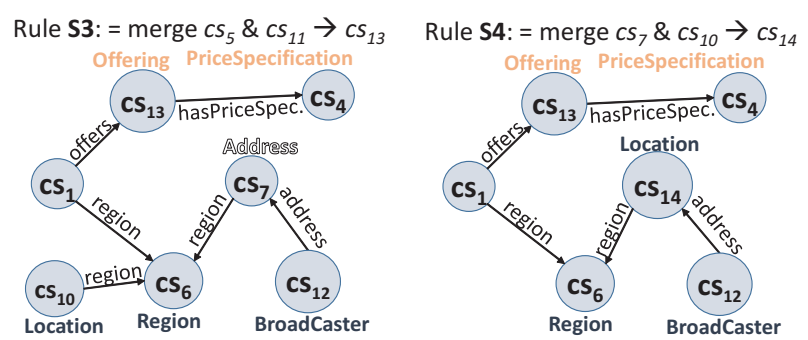

Figure 9: Example of merging CS's by using rules S3, S4

and $c s_{3}$ both derived their label offering from the Offering class of the GoodRelation ontology, according to $S 1$, they are merged into a new $\mathrm{CS}\left(c s_{11}\right)$. The references from/to $c s_{2}$ and $c s_{3}$ are also updated for $c s_{11}$. Besides, since the labels of $c s_{8}$ and $c s_{9}$ have Broadcaster as their non-general common ancestor in the ontology hierarchy, they are merged into $c s_{12}$ according to S2. The label of $c s_{12}$ is assigned by using the name of the common ancestor ontology class. The full description about updating the label of a CS after merging can be found in [17].

Structural merging. While semantic merging is a relatively safe bet, it may not always be applicable or effective enough to reduce the amount of merged CS's. Therefore, we also look at the structure of the CS's and their relationships to see if these can be merged. The idea here is to identify CS's that denote the same concept based on socalled "discriminative" properties, which are those with a high TF/IDF score (see the previous section). If the overlap between two CS's contains enough "discriminative" properties, we can merge them.

Figure 10 shows an example where the overlapping properties of $c s_{7}$ and $c s_{10}$ indicate that these CS's both originate from the "Location" entity. Here, the property rdfd: name is not discriminative since it appears in most of the CS's. However, properties rdfd:street-address and rdfd: region give evidence that both represent a "Location".

Equations 2 and 3 formally show the detailed computations for the TF/IDF score of each property in a cs and the cosine similarity score $\left(\operatorname{sim}_{i j}\right)$ between two cs's $\left(c s_{i}\right.$ and $\left.c s_{j}\right)$, respectively. In these functions total\#CSs is the total number of CS's, \#containedCSs $(p)$ is the number of CS having property $p$ in their property list.

$$
\begin{gathered}
t f i d f(p, c s)=\frac{1}{\left|D_{p}(c s)\right|} \times \log \frac{\text { total\#CSs }}{1+\# \text { containedCSs }(p)} \\
\operatorname{sim}_{i j}=\frac{\sum_{p \in\left(c s_{i} \cap c s_{j}\right)} t f i d f\left(p, c s_{i}\right) \times t f i d f\left(p, c s_{j}\right)}{\sqrt{\sum_{p_{i} \in c s_{i}} t f i d f\left(p_{i}, c s_{i}\right)^{2}} \times \sqrt{\sum_{p_{j} \in c s_{j}} t f i d f\left(p_{j}, c s_{j}\right)^{2}}}
\end{gathered}
$$

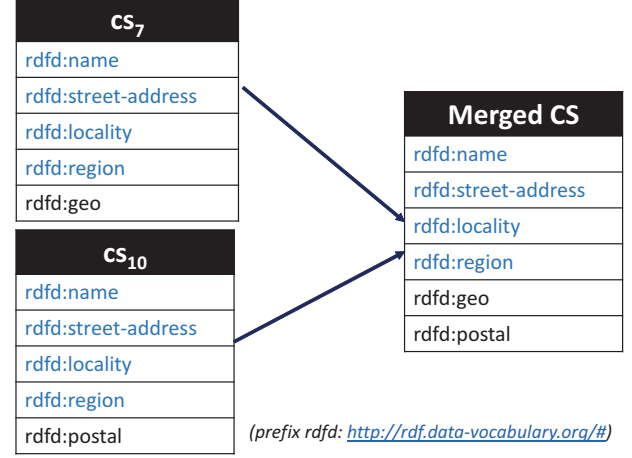

Figure 10: Merging CS's based on discriminative properties In addition to the set of properties in a CS, incoming relationship references from other CS's can also be used as an evidence in identifying similar CS's. Normally, a subject refers to only one specific entity via a property. For example, the property has_author of the subject "Book" always refers to an "Author" entity. Thus, if one CS, e.g., $c s_{1}$, refers to several different CS's e.g., $c s_{2}$ and $c s_{3}$, via a property $p$, this hints at $c s_{2}$ and $c s_{3}$ being similar.

In summary, two CS's are considered structurally similar if they are both referred from the same CS via the same property (rule $S 3$ ) or their property sets have a high TF/IDF similarity score (rule $S 4$ ). In Rule $3, \operatorname{ref}\left(c s, p, c s_{i}\right)$ is the number of references from $c s$ to $c s_{i}$ via property $p$, and $\tau_{i n f}$ is the infrequent threshold which is used to prevent nonfrequent references from being considered in applying the rule $S 3$. In Rule $4, \tau_{\text {sim }}$ is the similarity threshold above which we decide to merge two CS's.

RULE 3. If cs and $p$ exist with $\frac{r e f\left(c s, p, c s_{i}\right)}{f r e q(c s)}$ and $\frac{r e f\left(c s, p, c s_{j}\right)}{f r e q(c s)}$ greater than $\tau_{\text {inf }}$ then merge $c s_{i}$ and $c s_{j}$. (S3)

RULE 4. If the similarity score $\operatorname{sim}_{i j}$ between $c s_{i}$ and $c s_{j}$ is greater than $\tau_{\text {sim }}$ then merge $c s_{i}$ and $c s_{j}$. (S4)

Figure 9 shows the updates to the CS's and their relationships when we continue applying the rules $S 3$ and $S 4$. In this figure, $c s_{5}$ and $c s_{11}$ are merged according to the rule $S 3$ as they are both referred by $c s_{1}$ via the property offers. Besides, since $c s_{7}$ and $c s_{10}$ have high similarity score (as shown in Figure 10), they are merged into $c s_{14}$.

We experimentally observed that the best order of applying the rules for merging CS's is $S 1, S 3, S 2, S 4$. Further details can be found in [17].

\subsection{Schema Filtering}

Our goal is to represent a large portion of the input triples in a compact, human-friendly, relational schema. After CS merging, most of these merged classes ${ }^{12}$ cover a large amount of triples. However, it may happen that some classes still cover a limited number of RDF subjects, so if the merged

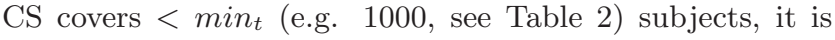
removed from the schema; and we limit the UML class diagram to the merged $U b_{t b l}$ CS's with highest frequency. Note that omitting CS's with low frequency will only marginally reduce overall coverage.

Preserving Dimension Tables. However, for this removal of classes (merged CS's) we make one exception, namely

\footnotetext{
12 At this stage, we also refer surviving merged CS's as classes,
} similar to UML classes. 
we conserve CS's that - although small in terms of covered subjects - are referred to many times from other tables. The rationale is that such CS's thanks to the large amount of incoming references represent important information of the dataset that should be part of the schema. This is similar to a dimension table in a relational data warehouse, which may be small itself, but is referred to by many millions of tuples in large fact tables over a foreign key. Thus, combining the information of basic CS detection and relationship detection, we preserve CS's with a high frequency of incoming references. However, detecting dimension tables should not be handled just based on the number of direct relationship references. The relational analogy here are snowflake schemas, where a finer-grained dimension table like CITY refers to an even smaller coarse-grained dimension table COUNTRY. To find the transitive relationships and their relative importance, we use the PageRank [16] algorithm on the graph formed by all CS's (vertexes) and relationships (edges, regardless of direction). In each iteration, the score of a merged CS is computed based on the references from other merged CS's and their scores computed in the previous iteration. Equation 4 shows the formula for each iteration:

$$
\begin{array}{r}
I R_{k}\left(c s_{i}\right)=\sum_{c s_{j} \rightarrow c s_{i}} I R_{k-1}\left(c s_{j}\right) \times \frac{r e f\left(c s_{j}, c s_{i}\right)}{\operatorname{refsTo}\left(c s_{i}\right)} \\
\times \frac{r e f\left(c s_{j}, c s_{i}\right)}{f r e q\left(c s_{j}\right)}+\operatorname{refsTo}\left(c s_{i}\right) \\
\operatorname{If}\left(I R_{k}\left(c s_{i}\right) \geq U b_{t b l}\right) \rightarrow c s_{i} \text { is a dimension } C S .
\end{array}
$$

The merged CS's having a score higher than a threshold $U b_{t b l}$ will be selected for inclusion in the schema. in which $I R_{k}\left(c s_{i}\right)$ is the indirect-referenced score of $c s_{i}$ after $k$ iterations, $\operatorname{ref}\left(c s_{j}, c s_{i}\right)$ is the number of references from $c s_{j}$ to $c s_{i}$, freq $\left(c s_{j}\right)$ is the frequency of $c s_{j}$, and refsTo $\left(c s_{i}\right)$ is the total number of direct references to $c s_{i}$.

Specifically, the number of iterations $k$ is set the same as the diameter of the CS graph. It is because, with that value, after $k$ iterations, the $I R_{k}$ score of each CS will get computed from all the CS's. To compute the diameter of the graph, we implemented a fast and simple algorithm described by [3].

Minimizing the number of infrequent properties. A final step of schema filtering considers eliminating CS properties, which as column in a relational table would have many NULL values. If the property coverage ratio (see Equation 5) is less than the infrequent threshold $\tau_{\text {inf }}$, that property is infrequent and it gets removed from the CS.

$$
\operatorname{coverageRatio}(p, c s)=\frac{f r e q(p, c s)}{f r e q(c s)}
$$

\subsection{Instance Filtering}

The output after labeling, merging, and schema filtering is a compact relational emergent schema. In the instance filtering phase, all RDF triples are visited again, and either stored in relational tables (typically $>90 \%$ of the triples, which we consider regular), or (the remainder) separately in a PSO table. Hence, our final result is a set of relational tables with foreign keys between them, and a single triple table in PSO format. In principle, the regular triples are those belonging to a merged CS (that survived schema filtering). However, not all such triples are considered regular in the end, as we perform three types of instance filtering, described next.
Maximizing type homogeneity. Literal object values corresponding to each property in a CS can have several different types e.g., number, string, dateTime. The relational model can only store a single type in each column, so in case of type diversity, a relational system like MonetDB must use multiple columns for a single property. They contain the type-cast value of the literal, if possible, and NULL otherwise. The number of columns needed for representing the data from a $c s_{i}$ hence is $\sum_{p \in c s_{i}} \#$ ofTypes $(p)$. This number can be large just due to a few triples having the wrong type (dirty data). To minimize the number of such columns, for each property, we filter out all the infrequent literal types (types that appear in $<\tau_{\text {inf }}$ percent of all instances). All triples of class instances with infrequent types are moved to the PSO table.

Relationship Filtering. We further filter out infrequent or "dirty" relationships between classes. A relationship between $c s_{i}$ and $c s_{j}$ is infrequent if the number of references from $c s_{i}$ to $c s_{j}$ is much smaller than the frequency of $c s_{i}$ (i.e., less than $\tau_{\text {inf }}$ percent of the CS's frequency). A relationship is considered dirty if the majority but not all the object values of the referring class (e.g., $c s_{i}$ ) refer to the instances of the referred class $\left(c s_{j}\right)$. In the former case, we simply remove the relationship information between two classes. In the latter case, the triples in $c s_{i}$ that do not refer to $c s_{j}$ will be filtered out (placed in the separate PSO table).

We note that in the general case of $n-m$ cardinality relationships, the relational model requires to create a separate mapping table that holds just the keys of both relations. However, in case one of the sides is $0 \ldots 1$, this is generally avoided by attaching a FK column to the table representing the other side. We try to optimize for this, by observing whether a multi-valued relationship is infrequent $\left(<\tau_{\text {inf }}\right)$. If so, we remove the excess relationship to the separate PSO table, such that all remaining subjects in the class have maximally one relationship destination. Finally, if almost all instances of one class have exactly one match in the other class but a few $\left(<\tau_{\text {inf }}\right)$ have none, we move all triples with that subject to the separate PSO table to preserve the exact $n$ - 1 cardinality (which keeps the FK column non-NULLable).

Multi-valued attributes. The same subject may have 0 , 1 or even multiple triples with the same property, which in our schema leads to an attribute with cardinality $>1$. While this is allowed in UML class diagrams, direct storage of such values is not possible in relational databases. Practitioners handle this by creating a separate table that contains the primary key (subject OID) and the value (which given literal type diversity may be multiple columns). The RDF bulk-loader of MonetDB does this, but only creates such separate storage if really necessary. That is, we analyze the mean number of object values (meanp) per property. If the meanp of a property $p$ is not much greater than 1 (e.g., less than $\left(1+\tau_{\text {inf }} / 100\right)$, we consider $p$ as a single-valued property and only keep the first value of that property in each tuple while moving all the triples with other object values of this property to the non-structured part of the RDF dataset. Otherwise, we will add a table for storing all the object values of each multi-valued property.

$$
\begin{aligned}
\operatorname{meanp}(p) & =\sum p(k) \times k \\
\text { where } p(k) & =\frac{\# \text { times } p \text { has } k \text { object values }}{\text { freq }(p)}
\end{aligned}
$$




\begin{tabular}{|l|c|c|}
\hline Parameter & Default & Description \\
\hline$U b_{t b l}$ & 1000 & number of tables upper bound \\
\hline $\min _{t}$ & 1000 & minimum table size \\
\hline$\tau_{i n f}$ & $5 \%$ & infrequent threshold \\
\hline
\end{tabular}

Table 2: Emergent Relational Schema Detection Parameters

\subsection{Parameter Tuning}

An important question that we needed to address is how the various parameters guiding the recognition process should be set. Choosing improper parameters might result in a "bad" final schema with e.g., small data coverage, lots of NULLs, etc. Further, since each input dataset can have different characteristics, it would be unfeasible to find a fixed parameter set that works optimally for all datasets.

The most dataset sensitive parameter we found to be the $\tau_{\text {sim }}$, used in labeling while matching ontologies using discriminative properties, as well as in the CS merging Rule 4 that determines up until which point merging should continue. It is a control on the strictness of finding equivalences between structures and ontologies, at 1 it is very strict while at 0 it is very lax. We evaluate the quality of the relational schema on two dimensions, namely (i) the number of tables (compactness of the schema) and (ii) its precision, which is the number of NOT-NULL cells, fill $(t)$, divided by the total number of cells, cap $(t)$, in all tables, as in Equation 7. There is a clear trade-off between having a compact schema and higher precision, depending on $\tau_{\text {sim }}$.

Our auto-tuned algorithm iteratively re-runs the labeling and merging steps with different values of $\tau_{\text {sim }}$. In each run, we measure the number of tables and the precision; we also compute a delta of these between successive values of $\tau_{\text {sim }}$. In Equation 8, $k$ is the total number of runs; $n T_{i}\left(n T n o m_{i}\right)$ and $\operatorname{prec}_{i}\left(\operatorname{prNom}_{i}\right)$ are the (normalized) number of tables and the schema precision at the $i^{\text {th }}$ run; nTdelta $a_{i}$ and $\operatorname{prDelta}_{i}$ are the relative change in the normalized number of tables and the precision at the $i^{\text {th }}$ run, respectively. We use the lowest value of $\tau_{\text {sim }}>0$ where $n$ Tdelta $a_{i}>$ prDelta $a_{i}$.

$$
\begin{gathered}
\text { prec }=\frac{\sum_{t} \operatorname{fill}(t)}{\sum_{t} \operatorname{cap}(t)} \\
n \operatorname{nnom}_{i}=\frac{n T_{i}-n T_{1}}{n T_{k}-n T_{1}} \quad \operatorname{prNom}_{i}=\frac{\text { prec }_{i}-\text { prec }_{1}}{\text { prec }_{k}-\text { prec }_{1}} \\
n \text { Tdelta } \\
\text { prDelta }_{i}=\text { nTnom }_{i}-n \text { prom }_{i-1} \\
\text { prom }_{i}-\text { prom }_{i-1}
\end{gathered}
$$

The left of Figure 11 shows normalized $n T_{i}$ and prec $_{i}$ for WebData Commons as a function of $\tau_{\text {sim }}$ in steps of 0.05 , while the right side shows the deltas between steps. Autotuning chooses the cross-over point of the deltas $\left(\tau_{-}\right.$sim $\left.=0.7\right)$.

\section{EXPERIMENTAL EVALUATION}

Metrics. We propose several metrics for evaluating the quality of the emergent schema. These metrics rely on the fact that a structure is considered to be good if it is compact (few and thin tables), precise (few NULLs) and has large coverage (few triples that have to be moved to separate PSO storage). Given an RDF dataset $R$ and its total number of triples $|R|$, the first performance metric, $C$, is the percentage of input triples covered by the schema:

$$
C=\frac{\sum_{1}^{n} \operatorname{cov}\left(t_{i}\right)}{|R|}
$$
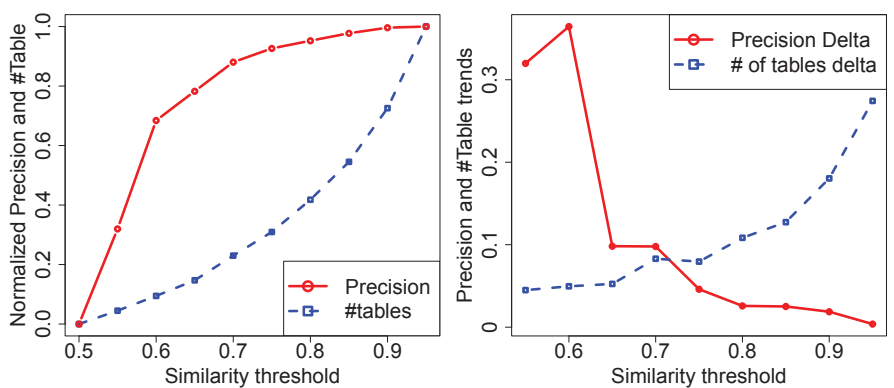

Figure 11: Left: $\tau_{\text {sim }}$ steps on X, \#Tables\&Precision on Y. Right: step deltas, auto-tuning selects cross-over (WebData)

\begin{tabular}{|l|l|l|}
\hline labels & WebData. & DBpedia \\
\hline top 3 & 3.6 & 3.8 \\
\hline final & 4.1 & 4.6 \\
\hline
\end{tabular}

Table 3: Human survey results on Likert scale

Each class in the structure is physically stored in a separate relational table. We define worth $w\left(t_{i}\right)$ of table $t_{i}$ as:

$$
\begin{aligned}
w\left(t_{i}\right)=\overbrace{\frac{\operatorname{cov}\left(t_{i}\right)}{\sum_{1}^{n} \operatorname{cov}\left(t_{i}\right)}}^{(I)} \times \overbrace{\left(\operatorname{prec}\left(t_{i}\right)+\frac{\operatorname{ref}\left(t_{i}\right)}{\sum_{1}^{n} \operatorname{ref}\left(t_{i}\right)}\right)}^{(I I)} \\
\text { where prec }\left(t_{i}\right)=\frac{\operatorname{fill}\left(t_{i}\right)}{\operatorname{cap}\left(t_{i}\right)}
\end{aligned}
$$

The precision $\operatorname{prec}\left(t_{i}\right)$ of the table $t_{i}$ is the fraction of nonNULL values in table $t i, \operatorname{cov}\left(t_{i}\right)$ is the number of RDF triples stored in $t_{i} ; n$ is the number of tables and re $f\left(t_{i}\right)$ is the number of FK's referring to $t_{i}$. Here, (II) sums the precision and the relative importance of the table considering the relationships between tables, while (I) denotes the contribution of the table for the coverage of the schema. As the schema is only compact if $n$ is small, the quality of the explored structure, $Q$, is defined as: $Q=\frac{\sum_{1}^{n} w\left(t_{i}\right)}{n}$.

\subsection{Experimental Results}

Labeling evaluation. We presented the emergent schemas of the DBpedia and WebDataCommons datasets to $19 \mathrm{hu}-$ mans and asked them to rate the labels. On a 5-point Likert scale from 1 (bad) to 5 (excellent) label quality, the top 3 labels of each table were scored by at least 3 persons. As shown in Table 3, the top 3 label candidates received an average rating of 3.6 for WebDataCommons and 3.8 for the DBpedia dataset. The finally chosen labels (one among the top 3 ) got better scores (4.1 and 4.6, respectively). We therefore conclude that the ordering of label candidates created by our algorithms produces encouraging results, as the chosen labels get higher ratings than the other candidates. Furthermore, our evaluation shows that 78\% (WebDataCommons) and $90 \%$ (DBpedia) of the labels are rated with 4 points or better, hence are considered "good" labels by the users. The emergent relational schemas for the nine datasets we tested are too large to include in this paper, Figure 12 shows EuroStat, one of the simpler schemas. ${ }^{13}$

Merging/Filtering performance. Figure 13 and Table 4 show the performance of the proposed merging algorithms and the filtering techniques for detecting a compact relational emergent schema with high coverage. According to

\footnotetext{
${ }^{13}$ See www.cwi.nl/ boncz/emergent for the other datasets.
} 


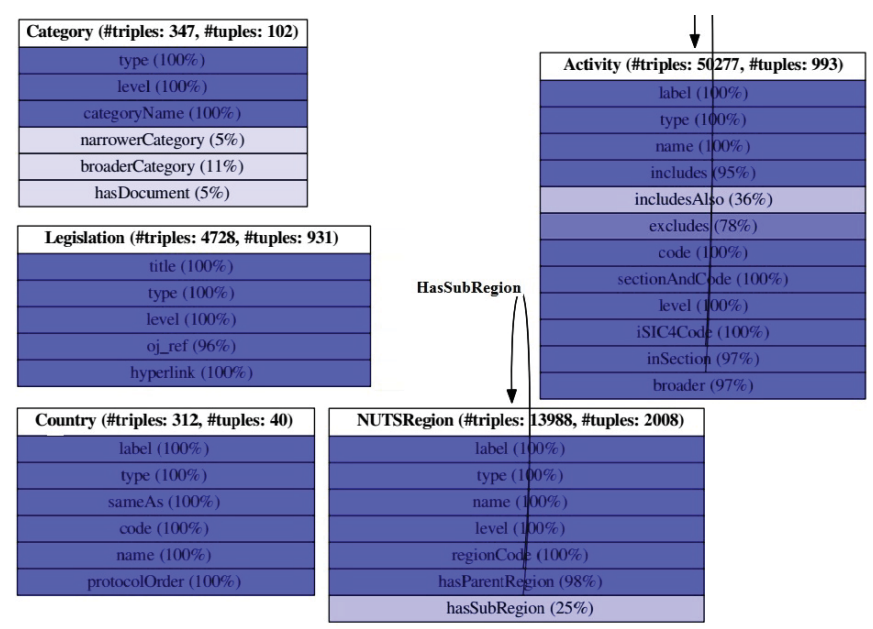

Figure 12: Final emergent schema for EuroStat - the lighter a column, the more NULLs (percentage in parentheses).

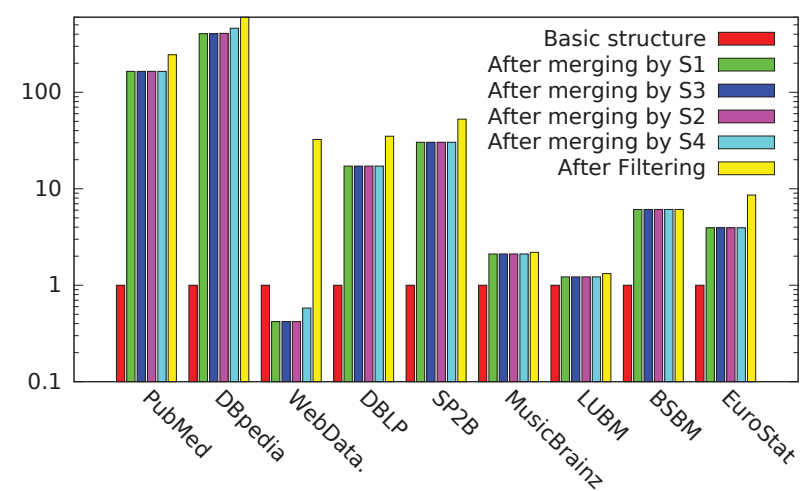

Figure 13: Schema quality $Q$ during merging \& filtering

Figure 13, the metric $Q$ of the explored structure, except for WebDataCommons, always increases after the merging and filtering steps. For WebDataCommons, the value of $Q$ decreases when merging CS's using rule S1. This stems from the fact that in WebDataCommons dataset each CS describing a certain entity such as Website may have many additional properties describing application attached to the website, and even use various properties for the same attribute (e.g., ogp.me/ns\#url, opengraphprotocol.org/schema/url, rdf . data-vocabulary.org/\#url for the website's URL), and thus, their merged CS's may contain properties with lots of NULLs values, causing the decrease of the metric $Q$. Nevertheless, the filtering step, by refining infrequent properties in the explored structure, can help addressing this issue and significantly increases the score of the metric $Q$. Comparing to the basic structure, the final schema of each experimental dataset is several orders of magnitude better in this metric.

Table 4 also shows that after the schema filtering, the final schema in all cases achieves very high coverage. We see that synthetic RDF benchmark data (BSBM, SP2B, LUBM) is fully relational, and also all dataset with nonRDF roots (PubMed, MusicBrainz, EuroStat) get $>99 \%$ coverage. Most surprisingly, the RDFa data that dominates WebDataCommons and even DBpedia is more than $90 \%$ regular. Further, a non-complete manual inspection of the $<10 \%$ irregular triples in these datasets appeared to show mainly mistyped properties, so our suspicion is that much of this irregularity is in fact data "dirtiness".

Computational cost. Figure 14 shows that the time for detecting the emerging schema is negligible compared to

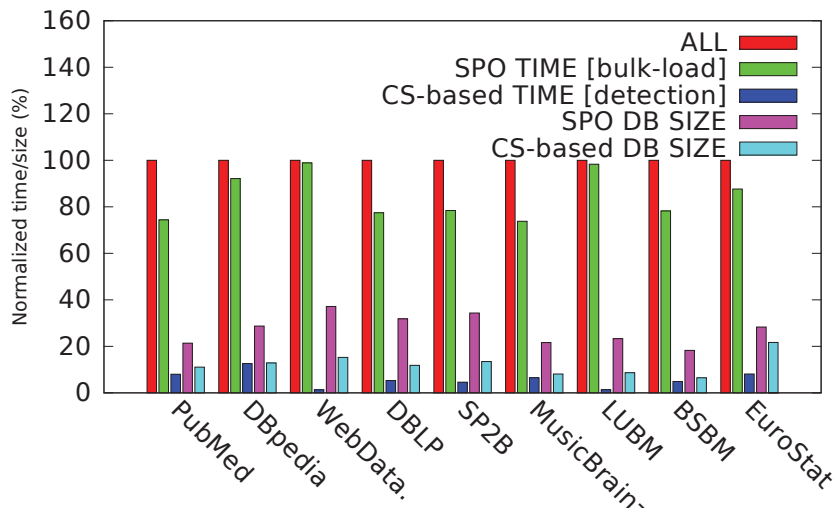

Figure 14: Building time \& database size for single triple table (SPO) and reorganized relational tables (CS-based) (normalized by bulk-load time and database size for all six S,P,O table permutations (ALL)).

\begin{tabular}{|c||r|r|r|r|r|r|}
\hline \multicolumn{1}{|c||}{} & \multicolumn{3}{c|}{ Number of tables } & \multicolumn{3}{c|}{ Coverage - Metric $C(\%)$} \\
\hline Datasets & $\begin{array}{c}\text { before } \\
\text { merging }\end{array}$ & $\begin{array}{c}\text { after } \\
\text { merging }\end{array}$ & $\begin{array}{r}\text { remove } \\
\text { small } \\
\text { tables }\end{array}$ & $\begin{array}{r}\text { remove } \\
\text { small } \\
\text { tables }\end{array}$ & $\begin{array}{c}\text { prune } \\
\text { infreq. } \\
\text { prop. }\end{array}$ & $\begin{array}{c}\text { final } \\
\text { schema }\end{array}$ \\
\hline LUBM & 17 & 13 & 12 & 100 & 100 & $\mathbf{1 0 0 . 0 0}$ \\
\hline BSBM & 49 & 8 & 8 & 100 & 100 & $\mathbf{1 0 0 . 0 0}$ \\
\hline SP2B & 554 & 13 & 10 & 99.99 & 99.65 & $\mathbf{9 9 . 6 5}$ \\
\hline MusicBrainz & 27 & 12 & 12 & 100 & 99.9 & $\mathbf{9 9 . 6 0}$ \\
\hline EuroStat & 44 & 10 & 5 & 99.73 & 99.53 & $\mathbf{9 9 . 5 3}$ \\
\hline DBLP & 249 & 9 & 6 & 100 & 99.68 & $\mathbf{9 9 . 6 0}$ \\
\hline PubMed & 3340 & 14 & 12 & 100 & 99.75 & $\mathbf{9 9 . 7 3}$ \\
\hline WebData. & 13354 & 3000 & 253 & 98.17 & 94.37 & $\mathbf{9 2 . 7 9}$ \\
\hline DBpedia & 439629 & 542 & 234 & 99.12 & 96.68 & $\mathbf{9 5 . 8 2}$ \\
\hline
\end{tabular}

Table 4: \#tables and metric $C$ after merging \& filtering

bulk-loading time for building a single SPO table as well as building all the six permutations of S, P, O (marked [ALL]). Thus, MonetDB integrates emergent schema detection into its RDF bulk-loading without recognizable delay.

Compression. Figure 14 shows that the database size stored using relational tables can be two times smaller than the database size of a single SPO triple table. The reason is that in the relational representation the $\mathrm{S}$ and $\mathrm{P}$ columns effectively get compressed away, only the 0 columns remain.

Query processing. As a proof that the recognized emergent schema can be easily integrated and boost the performance of existing RDF stores, we report on the effort at OpenLink to integrate emergent relational schema technology in one of the state-of-the-art RDF stores, Virtuoso [8] ${ }^{14}$ It was a few months work to integrate Characteristic Set based storage, query execution and query optimization in Virtuoso. We compare a classic Virtuoso RDF quad table (Virt-Quad) and this CS-based implementation (Virt-CS) on the BSBM benchmark at 10 billion triples scale.

The experimental results in Table 5 show that exploiting the emergent relational schema even in this very preliminary implementation already improves the performance of Virtuoso on a number of BSBM Explore queries by up to a factor of 5.8 (Q3, Hot run). Note that the Cold run is much slower comparing to the Hot run as most of the time goes in the statistics gathering, not in the execution. We see less gain from CS's in other queries, e.g., Q5, since the first condition on the BSBM products (on a range of numeric property) is selective, so the other columns of the CS (or self-joins to

\footnotetext{
${ }^{14}$ https://github.com/v7fasttrack/virtuoso-opensource
} 


\begin{tabular}{|c|c|c|c|c|c|c|c|c|c|c|c|c|c|c|c|}
\hline \multirow[b]{2}{*}{ RDF Store } & \multicolumn{3}{|c|}{ Q2 } & \multicolumn{3}{|c|}{ Q3 } & \multicolumn{3}{|c|}{ Q5 } & \multicolumn{3}{|c|}{ Q7 } & \multicolumn{3}{|c|}{ Q8 } \\
\hline & Cold & Hot & Opt. & Cold & Hot & Opt. & Cold & Hot & Opt. & Cold & Hot & Opt. & Cold & Hot & Opt. \\
\hline Virt-Quad & 11567 & 7 & 4.2 & 4210 & 53 & 40.2 & 3842 & 1350 & 18.6 & 19401 & 9 & 5.3 & 14644 & 9 & 4.4 \\
\hline Virt-CS & 2485 & 6 & 3.5 & 2965 & 9 & 5.4 & 2130 & 712 & 4.2 & 11642 & 6 & 4.5 & 5370 & 5 & 3.3 \\
\hline
\end{tabular}

Table 5: Query time (msecs) w/wo the recognized schema

(Cold: First query runtime after re-starting the server; Hot: Run the query 3 times and get the last runtime; Opt.: Query optimization time)

RDF quads) are done on a small fraction of the subjects of the first range check. In Q3 more single-valued properties are accessed per subject, resulting in much more gain.

By collapsing multiple triple patterns into a single abstract CS table, query optimization gets a plan search space of the same order as for the equivalent SQL. For Q3, the compilation time drops from 40.2 msecs to 5.4 msecs when using the recognized schema. In many RDF applications, e.g. Open PHACTS ${ }^{15}$, query optimization time dominates and can run into the tens of seconds. Due to the extreme search space resulting from triple patterns, there are often ad hoc restrictions on plans, e.g. no hash join or no joins on hash build sides. With CS, a more thorough search of the plan space becomes again practicable and we expect qualitatively better plans to result.

\section{RELATED WORK}

We note that previous work has already proposed building relational-resembling RDF stores $[22,7,12,21,4]$. However, these proposals either demand the presence of an allexplaining ontology (which then gets remapped to relational tables), or ask the database system administrator to create and maintain "property tables" explicitly. Our approach, in contrast, does not require any form of explicit schema ingestion. Second, since these approaches just use the structure internally in the SPARQL engine to make things faster, they do not address the challenge of making the schema understandable to humans (compactness, finding short aliases). For the latter, a related line of work is creating summaries of the graph structure to aid query formulation [6], yet these do not focus on making RDF database systems faster, and typically require a cluster to compute, whereas our approach is cheap and can piggyback on RDF bulk-loading. Related to the automatic structure exploration from data is work on ontology mining [13] which discovers ontologies from unstructured text on the web. In our approach, we recognize the emergent structure in RDF data (e.g., mixing of ontologies), and do not change the semantics, and focus on providing a relational view of it.

Frequent itemset mining, which has been studied in many data mining papers $[1,5,9]$, is equivalent to the basic CS recognition, originally proposed by [14]. We use this technique but go beyond that by finding a schema graph with cross-CS relationships, and we employ a host of techniques to make this schema graph compact and human-friendly (finding labels).

A recent study on the structure refinement for the RDF data, [2] proposed an integer linear programming (ILP)based algorithm which allows an RDF dataset being partitioned into a number of "sorts" where each sort satisfies a predefined structured-ness fitting threshold. This approach, relying mainly on the similarity and correlation between the properties of sorts, may merge subjects describing unrelated entities but having many common properties into a single sort (as also shown in their experiment with Drug Com-

\footnotetext{
${ }^{15} \mathrm{http://www.openphacts.org/}$
}

pany and Sultan), while our solution only merges related CS's together by exploiting the discriminating properties and the availability of the semantics/ontologies information. Besides, no relationship exploration as well as labeling for the sorts are considered in this work, and thus, no relational schema is recognized.

Consulting external resources for entity labeling is suggested by [20] in the context of table data reconstruction as well as by [19] a study on labeling hierarchical clusters. The former study shows that column names and table names usually cannot be found in table data itself. To reconstruct HTML table data they therefore rely on an external database with hyponym information. The latter study also mentions that documents often do not contain selfdescriptive terms. To overcome this, they suggest using "anchor texts" as an additional resource in their document labeling task. Anchor texts are pieces of text on and next to hyperlinks to a specific document. The relational equivalent of anchor texts are names of foreign key relationships. In our case we rely on property names that refer to other tables for name suggestions, supplemented by ontology information when present.

\section{CONCLUSIONS}

In this paper, we introduced the notion of - and demonstrated practical techniques for - discovering an emergent relational schema in RDF datasets, that recovers a compact and precise relational schema with high coverage and useful labels as alias for all machine-readable URIs (which it preserves). The functional benefit of an emergent relational schema for RDF datasets is both in giving users better understanding of the structure of an RDF dataset, while also allowing the often $>90 \%$ of regular triples to be queried from existing SQL applications, which still dominate the IT industry. Our MonetDB RDF bulk loader enables this. We think that this also provides impetus to make SQL more semantic, e.g. stimulating usage of URIs in SQL metadata.

The emergent relational schema can also be used under the cover of an SPARQL engine as a new storage approach, where the $90 \%$ regular triples are stored in tabular structures and the rest in SPO format. We think that the knowledge of an emergent schema gives SPARQL engines just what they need to close the performance gap with SQL systems. This we demonstrated in Virtuoso, with gains both in compression, query execution and query optimization. The tabular structure opens up many opportunities to improve physical access patterns using (partial) clustered indexes, zone maps, table partitioning and even database cracking [11].

Looking ahead, the prospect of people supporting SQL applications on top of RDF data raises many new questions. Users will desire to tweak a found emergent schema by hand, e.g. by manually improving some labels. We propose making a found emergent schema explicit using a vocabulary, and researching techniques to control schema evolution to preserve schema stability while the emergent schema adapts over time to changes in the underlying RDF datasets. 


\section{REFERENCES}

[1] R. Agrawal et al. Fast algorithms for mining association rules. In $V L D B, 1994$.

[2] M. Arenas et al. A principled approach to bridging the gap between graph data and their schemas. In $V L D B$, 2014.

[3] K. Boitmanis et al. Fast and simple approximation of the diameter and radius of a graph. In Experimental Algorithms, pages 98-108. Springer, 2006.

[4] A. Bornea et al. Building an efficient RDF store over a relational database. In SIGMOD, 2013.

[5] D. Burdick et al. Mafia: A maximal frequent itemset algorithm for transactional databases. In ICDE, 2001.

[6] S. Campinas et al. Introducing RDF graph summary with application to assisted SPARQL formulation. In DEXA Workshops, 2012.

[7] E. Chong et al. An efficient SQL-based RDF querying scheme. In $V L D B, 2005$.

[8] O. Erling. Virtuoso, a hybrid RDBMS/graph column store. IEEE Data Eng. Bull, 2012.

[9] K. Gouda and M. Zaki. Efficiently mining maximal frequent itemsets. In $I C D M, 2001$.

[10] A. Gubichev and T. Neumann. Exploiting the query structure for efficient join ordering in SPARQL queries. In EDBT, 2014.

[11] S. Idreos, M. L. Kersten, and S. Manegold. Database cracking. In Conference on Innovative Data Systems Research (CIDR), pages 68-78, Asilomar, California, 2007.
[12] J. Levandoski and M. Mokbel. RDF data-centric storage. In $I C W S, 2009$.

[13] Y. Li et al. Mining ontology for automatically acquiring web user information needs. KDE, 2006.

[14] T. Neumann and G. Moerkotte. Characteristic sets: Accurate cardinality estimation for RDF queries with multiple joins. In ICDE, 2011.

[15] R. Neumayer et al. When simple is (more than) good enough: Effective semantic search with (almost) no semantics. In Advances in Information Retrieval. Springer, 2012.

[16] L. Page et al. The pagerank citation ranking: Bringing order to the web. Technical report, Stanford, 1999.

[17] L. Passing. Recognizing, naming and exploring structure in RDF data. Master's thesis, Technische Universität München, 2014.

[18] G. Salton and M. J. McGill. Introduction to modern information retrieval. 1983

[19] P. Treeratpituk and J. Callan. Automatically labeling hierarchical clusters. In DGSNA, 2006.

[20] P. Venetis et al. Recovering semantics of tables on the web. In $V L D B, 2011$.

[21] Y. Wang et al. Flextable: using a dynamic relation model to store RDF data. In DASFAA, 2010.

[22] K. Wilkinson. Jena property table implementation. Technical report, HP Labs, 2006. 